\title{
COLOSTOMÍA HÚMEDA EN DOBLE BARRA: ANÁLISIS DE UNA DERIVACIÓN
}

\author{
A. BLANCO DÍEZ, E. FERNÁNDEZ ROSADO, L. ÁlVAREZ CASTELO, J. SÁNCHEZ \\ RODRÍGUEZ-LOSADA, V. CHANTADA ABAL, S. NOVÁS CASTRO, A. BARBAGELATA \\ LÓPEZ, J. SERRANO BARRIENTOS, M. GONZÁLEZ MARTÍN
}

Servicio de Urología. Hospital Juan Canalejo. La Coruña.

Actas Urol Esp. 27 (8): 611-617, 2003

\section{RESUMEN}

COLOSTOMÍA HÚMEDA EN DOBLE BARRA: ANÁLISIS DE UNA DERIVACIÓN

En este artículo revisamos y analizamos nuestra experiencia en la realización de la colostomía húmeda o urocolostomía presentando siete casos. Se trata de: tres mujeres a una de las cuales se le practicó una urocolostomía por un mielomeningocele con incontinencia total de heces y vejiga neurógena, a otra por una neoplasia ginecológica tras exenteración pélvica, $\mathrm{y}$ a la tercera debido a una cistoproctitis rádica tras radiación por neoplasia ginecológica. Cuatro hombres de los cuales en uno se realizó urocolostomía por una paraplejia con múltiples fístulas urinarias y vejiga neurógena y tres tras exenteraciones pélvicas por neoplasias (en dos casos vesicales y en otro caso de sigma con infiltración vesical). Los resultados fueron buenos durante el seguimiento de todos los casos no habiéndose reportado casos de alteraciones metabólicas ni pielonefritis. En todos los casos representó una buena opción terapéutica para estos pacientes.

PALABRAS CLAVE: Colostomía húmeda. Derivación urinaria. Calidad de vida.

\section{ABSTRACT}

DOUBLE BARRED WET COLOSTOMY: ANALYSIS

We analise our experience in performing the wet colostomy, also called urocolostomy and present seven cases treated at our hospital in which this was used. We present: three women, one underwent an urocolostomy as a result of a mielomeningocele with urinary and fecal incontinence, other because a pelvic malignancy, and the third after developing a post radiotherapy cistoproctitis. Four men, one underwent this surgical procedure as a result of a traumatic paraplejia with multiple urinary fistulae and neurogenic bladder, the other three were secondary to pelvic malignancies (two bladder and one sigmoid malignacies). The outcome was fine in all cases with no pielonefritis neither metabolic disbalances, in all cases it represented a good option for these patients.

KEY WORDS: Wet colostomy. Urinary diversion. Quality of life.

$\mathrm{L}^{\mathrm{a} a \mathrm{sen}}$ a colostomía húmeda en doble barra es una técnica quirúrgica de escaso uso, y por tanto poco conocida. Sin embargo puede ser de utilidad en cirugía de exenteración pélvica o en otros casos en los que nos sea imposible mantener la anatomía de los tramos bajos urinario y digestivo. Tiene varias ventajas, entre las que destacan la mayor sencillez de la técnica en comparación con la derivación doble, así como la mejor calidad de vida que ofrecemos al paciente. Por otra parte la derivación de los uréteres al asa distal semiaislada disminuye la longitud de contacto con la mucosa colónica y subsecuentemente las alteraciones metabólicas. Además con esta técnica se 
evita casi en su totalidad el contacto de la orina con material fecaloideo por lo que se omite el riesgo de infección del tramo urinario superior, y la diarrea acuosa. Su utilización está condicionada a la patología de base, a las características del paciente, al resultado que se quiere conseguir a corto, medio o largo plazo, y a la experiencia y conocimiento del cirujano. Además, en muchas ocasiones, el problema surge justo en el momento de la cirugía, con escaso tiempo para decidir sobre el tipo de solución a un problema inesperado que impide el uso de las derivaciones más comúnmente conocidas.

Por todo ello decidimos revisar y analizar siete casos en los que fue empleada tanto para no olvidar su existencia como para analizar su funcionamiento, sus complicaciones y la repercusión junto a la calidad de vida que ofreció a cada uno de los pacientes.

\section{MATERIAL Y MÉTODOS}

Se presentan siete casos en los que se utilizó esta derivación.

Caso $n^{\circ} 1$ : Mujer de 31 años con antecedentes personales de mielomeningocele congénito corregido quirúrgicamente en la infancia. Importantes secuelas motoras. Vejiga neurógena e incontinencia de heces. A los 13 años se le realizó una urocolostomía como solución a sus problemas de incontinencia. El seguimiento supera los 20 años. Durante los 10 primeros años la urocolostomía se mantuvo normofuncionante, con función renal normal $(\mathrm{Crp}=0,9)$. Tanto las UIV como las ecografías no mostraron alteraciones. Tan solo se observó un ingreso hospitalario en relación con una pielonefritis aguda unilateral que se resolvió con antibióticos. La paciente presentó, debido a su vejiga desfuncionalizada, un episodio de piocisto por Pseudomona aeruginosa que se resolvió con antibioterapia. Este mismo proceso ocurrió un año después y ante la situación de un piocisto recidivante se decidió su manipulación quirúrgica, practicando mediante resección transuretral una amplia fístula vesico-vaginal desde el centro del retrotrígono. En revisiones posteriores se comprobó la permanencia de dicha fístula con bajo débito de liquido claro y posteriormente de débito nulo. Durante los siguientes años no se observaron infecciones urinarias. Tanto las UIV como las ecografías fueron normales, excepto una discreta dilatación pielocalicial bilateral intermitente (no observada en todas las revisiones), en relación probable a diuresis momentánea u ocupación de la colostomía y salida de los orificios ureterales por heces en el asa de colon. Actualmente la paciente está asintomática, con función renal normal $(\mathrm{Crp}=1)$, UIV y ecografías normales sin datos de reflujo ni dilatación ureteral, con buena adaptación a la única bolsa de urocolostomía, y sin datos de infecciones urinarias. No hemos observado alteraciones metabólicas derivadas de la derivación urinaria durante estos años. Sus únicos problemas actuales residen en el sobrepeso y pies zambos, no corregidos quirúrgicamente por los problemas motores asociados. La paciente tiene en la actualidad 31 años.

Caso $n^{\circ}$ 2: Mujer de 30 años con antecedentes personales de carcinoma de cérvix operado mediante histerectomía radical (Wertheim). La anatomía patológica demostró un carcinoma epidermoide que infiltraba cérvix, útero, vagina y parametrio, con bordes quirúrgicos negativos, y linfadenectomía positiva. Posteriormente recibió quimioterapia y radioterapia externa más braquiterapia endocavitaria. Un año después la paciente consulta en nuestro Servicio por dificultad miccional y retenciones urinarias que precisan autocateterismos intermitentes cada 8 horas. Diagnosticada de recidiva tumoral con afectación de recto y vejiga se le practica una cistectomía radical más amputación abdominoperineal, vaginectomía y urocolostomía. La anatomía patológica demostró infiltración por carcinoma epidermoide de vejiga y recto. Año y medio después la paciente falleció por metástasis generalizadas. Durante el corto seguimiento la urocolostomía se mantuvo normofuncionante, con función renal normal (Crp=1), UIV y ecografías normales sin datos de reflujo ni dilatación ureteral, y sin infecciones urinarias-pielonefritis reseñables, así como ausencia de alteraciones metabólicas.

Caso $n^{\circ}$ 3: Varón de 67 años, con antecedentes personales de carcinoma vesical de células 
transicionales estadio IV-A, e instilaciones endovesicales con BCG. Tres años después se le practica adenomectomía retropúbica detectándose foco microscópico de adenocarcinoma de próstata Gleason IV (PSA 2,9). El paciente ingresa en el Servicio de Cirugía General por estreñimiento y rectorragias diagnosticándose un tumor estenosante de recto. En el TAC se observa gran tumoración que afecta a próstata, suelo vesical, sigma y recto con hidronefrosis bilateral. La gammagrafía ósea es normal. Se practica una cistoprostatectomia radical con amputación abdomino-pélvica y urocolostomía. La anatomía patológica demuestra un carcinoma de células transicionales estadio IV-C que infiltra tejido adiposo perirectal, vesículas seminales y próstata, con implantes peritoneales y ganglios ilíacos positivos. Tras recuperarse de la cirugía, en la primera revisión se le remite al Servicio de Oncología para tratamiento neoadyuvante. Posteriormente perdemos el seguimiento de este paciente.

Caso nำ 4: Varón de 76 años con antecedentes personales de infecciones urinarias de repetición desde hacía un año resueltas con tratamiento antibiótico. Consulta en nuestro servicio por disminución de peso, neumaturia y fecaluria. Al tacto rectal se evidencia una masa. En el enema opaco y en la rectosimoidoscopia se visualiza un tumor estenosante. La biopsia realizada demuestra que se trata de un adenocarcinoma de sigma. Una ecografía demuestra impronta en la pared posterior vesical y en el TAC una masa en sigma que infiltra la pared posterior vesical con presencia de gas en su interior, y fístula vésico-intestinal. Se realiza cistoprostatectomía radical con resección de sigma y urocolostomía. La anatomía patológica demuestra un adenocarcinoma de sigma que invade vejiga con bordes quirúrgicos libres y ganglios negativos. El paciente se remite al Servicio de Oncología donde se administra radioterapia y quimioterapia. El seguimiento actual de este paciente es de un año. En la última revisión la UIV no muestra alteraciones significativas. La urocolostomía se mantiene normofuncionante. La función renal en normal $(\mathrm{Crp}=0,9)$. No se observan datos de infecciones urinarias-pielonefritis. La adaptación a la bolsa de urocolostomía es buena. No hay alteraciones metabólicas.

Caso $n^{\circ}$ 5: Varón de 82 años con antecedentes de lobectomía pulmonar 20 años antes. Carcinoma vesical infiltrante a tratamiento paliativo con resecciones transuretrales, quimioterapia con epirubicina y radioterapia, desde 1988. Tras sufrir una obstrucción intestinal de origen tumoral se le realiza una colostomía. Más adelante desarrolla una fístula recto vesical por lo cual se interviene realizándose anastomosis ureteral bilateral a colon y cerrándose el muñón fistuloso distal. Fue dado de alta perdiéndose el seguimiento.

Caso $n^{\circ}$ 6: Varón 35 años. Accidente de tráfico en 1992. Tetraplejia por fractura c7 d1 con sección medular. Múltiples fracturas, entre ellas tibia y peroné derechos, y rama isquiopubiana izquierda. Paciente que acude a su última revisión en la Unidad de Lesionados Medulares de nuestro centro en 1994, por importante problema social. Ingresa en nuestro centro en mayo de 2002 con pésimo estado general. Asocia: Infección del tracto urinario, úlceras por presión grado IV en isquion izquierdo y derecho, varias fístulas uretro-escrotales, anquilosis de caderas y rodillas, múltiples fístulas urinarias a periné $\mathrm{y}$ nalgas. Se realizan varios desbridamientos y curas por cirugía plástica de sus úlceras por presión. Ante la existencia de múltiples fístulas urinarias a periné y nalgas que no remiten tras sondaje vesical se realiza en julio de 2002 una cistostomía asociándose una colostomía de descarga. Durante los siguientes meses persisten las infecciones de orina y las fístulas urinarias por lo cual en octubre se realiza urocolostomía abocando uréteres a muñón distal de colostomía previa. Actualmente tras 4 meses de seguimiento el paciente se encuentra sin fístulas urinarias, con una mejoría clínica evidente y un estado general muy bueno dentro de su situación.

Caso nํ 7: Mujer 53 años. Trombosis venosa profunda y tromboembolismo pulmonar. Filtro en vena cava. Carcinoma de cérvix tratado con radio- 
terapia pélvica y cirugía de rescate cuya anatomía patológica se define como "no tumor residual". Ingresa por cuadro de dolor y tenesmo rectovesical, e infección de orina. En Resonancia Nuclear Magnética: ureterohidronefrosis bilateral por compresión de uréteres, no recidiva neoplásica. Cistoscopia: fístula vésico-vaginal. Colonoscopia: fístula recto-vaginal. El 8-8-02 se realiza colostomía húmeda en doble barra. Última revisión en Abril 03 asintomática. Urografía intravenosa (Fig. 3): no dilatación pieloureteral.

\section{DISCUSIÓN}

Afortunadamente en escasas ocasiones nos encontramos con pacientes que requieran derivación urinaria y fecal, siendo en la mayoría de los casos neoplasias pélvicas que requieren una exenteración completa. En estos raros casos tenemos que sopesar el estado del paciente y su calidad de vida así como valorar el ofrecer la mínima comorbilidad asociada a la cirugía a la hora de ofertar un procedimiento. Parece lógico que la posibilidad de abocar la orina y las heces a una misma bolsa a través de un único estoma facilita la técnica, y también disminuye la morbilidad postoperatoria y mejora la calidad de vida posterior. Sin embargo esto también posee sus complicaciones y durante años fue una solución poco apreciada por el cirujano.

Los pioneros en la idea de la evacuación de orina $\mathrm{y}$ heces por un único conducto en la reconstrucción tras exenteraciones pélvicas fueron Brunschwig y Daniel ${ }^{1,2}$, en los años 50 y 60. La solución que ellos idearon consistía en colostomía terminal y anastomosis ureterocolónicas directas a cada lado. Esta técnica no evitaba el reflujo de orina infectada, con las consecuentes pielonefritis de repetición. Asimismo las importantes alteraciones electrolíticas, así como la diarrea acuosa asociada a la mezcla de orina y heces en el colon, también contribuyó a que dicha técnica fuese abandonada por la mayor parte de los cirujanos, los cuales se decantaron por el uso de dos estomas separados cuando era necesaria la derivación urinaria y fecal. Es en 1989 cuando Carter, Dalton y Garnett, describen la nueva técnica de la colostomía húmeda en doble barra, aportando tres casos con seguimiento de 3, 13 y
18 meses $^{3}$. Esta técnica consiste en realizar la colostomía en barra dejando un muñón distal a la misma y cerrado en su porción distal, sobre el cual se realiza la anastomosis ureterocolónica con técnica antirreflujo. Los mismos autores presentan en 1994 una serie con 11 casos y un buen seguimiento a largo plazo. En esta serie se observa que todos los pacientes estuvieron satisfechos con un solo estoma, casi todos experimentaban salida continua de orina debido a movimiento intestinales peristálticos. Se estudió el residuo en el muñón colónico observándose menor de 25cc, y ausencia de contaminación importante. Aunque existía bacteriuria en casi todos los conductos, no hubo casos de pielonefritis clínica ni deterioro de función renal por pielonefritis crónica o reflujo. No existieron alteraciones electrolíticas en ningún caso. Solo hubo un caso de hidronefrosis por estenosis de la anastomosis ${ }^{4}$. Si aceptamos que la definición de eficacia de una derivación urinaria consiste en su capacidad para mantener la integridad del tracto superior, y la ausencia de alteraciones electrolíticas parece que esta técnica podría atender dichos requerimiento como asimismo se puede extraer de los datos de nuestra serie.

Esta derivación puede ser utilizada en cualquier caso en que sea deseable una derivación urinaria y fecal cómoda y de fácil ejecución evitando la realización de anastomosis intestinales. Por tanto no solo estaría indicada en cirugía de exenteración pélvica por tumores malignos, sino también en patología benigna, por ejemplo pacientes tetrapléjicos con vejiga neurógena e incontinencia fecal como los descritos en nuestra serie y en otros estudio, pacientes con cistitis/ proctitis rádicas intratables ${ }^{5}$, etc.

La técnica de colostomía en doble barra, tiene por sí misma ciertas ventajas en comparación a la urocolostomía clásica, además de disminuir el riesgo de pielonefritis por reflujo y por la mezcla de heces y orina, disminuye también el riesgo de una temida y sin embargo poco estudiada complicación: la aparición de cáncer de colon que se asocia más frecuentemente a la mezcla de la corriente de excrección fecal y urinaria ${ }^{6,7}$. Por otra parte no todos los cirujanos están de acuerdo en que un estoma en "loop" como el de la uro- 
colostomía evite totalmente el ascenso de bacterias hacia tramos urinarios superiores, y lo que es más importante el papel que puede jugar esa colonización bacteriana del asa distal, ya comentada antes, lógica, y en principio con escasa correlación clínica, en el desarrollo de carcinoma de colon en asa distal ${ }^{8,9}$. Por tanto, podemos concluir que no esta claro que la separación de las dos corrientes eviten del todo la aparición de cáncer de colon ${ }^{10,11}$.

Un concepto también importante a tener en cuenta durante este tipo de intervención es la realización de una correcta técnica antirreflujo, ya que como se ha comentado aunque no haya mezcla urinario-fecaloidea si existe colonización bacteriana en el asa distal. Por tanto dicha cirugía antirreflujo durante la anastomosis ureterocolónica es fundamental para evitar la pielonefritis ${ }^{12}$.

En lo que se refiere a las alteraciones hidroelectrolíticas se observan de forma escasa con esta técnica ya que el contacto de la orina con la mucosa del colon es mínimo siempre y cuando el muñón distal hacia el que van abocados los uréteres sea corto, si esto es así la absorción de orina será mínima así como los problemas iónicos ${ }^{13}$.

En lo referente a nuestra serie, la urocolostomía fue empleada en siete distintos casos: una vejiga neurógena con incontinencia de heces secundaria a un mielomeningocele congénito; un carcinoma de cérvix recidivado que invadía recto y vejiga; dos carcinomas de células transicionales de vejiga que invadían recto-sigma; un adenocarcinoma de sigma que invadía vejiga, y un paciente tetrapléjico con múltiples fístulas urinarias, y una cistoprostitis rádica tras radiación por neoplasia ginecológica.

En la técnica quirúrgica se empleo anastomosis independiente de los uréteres en borde antimesentérico del colon, realizando túnel submucoso antirreflujo y cateterizando los uréteres con sondas en "J". Se realizó urocolostomía en "doble barra" con lo que se consigue la eliminación de heces y orina de manera independiente. (Figs. 1, 2 y 3).

Como complicaciones posibles se encontrarían el reflujo ureteral, la estenosis de las anastomosis uretero-cólicas, y las infecciones urinariaspielonefritis ascendentes.

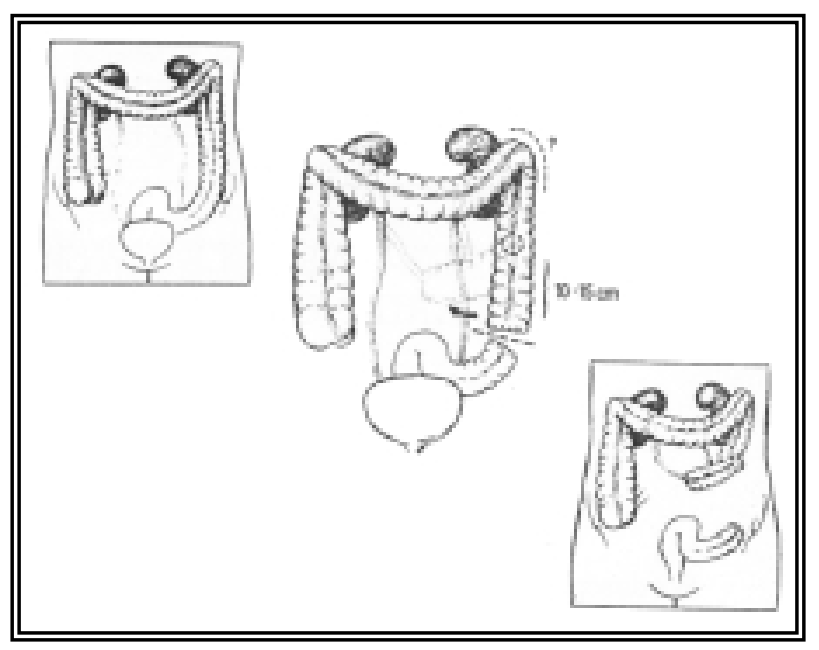

FIGURA 1. Técnica quirúrgica de urocolostomia. Extraída de J Urol 1994; 152: 2312-23154.

En ninguno de los casos tras el seguimiento, y después de revisar las pruebas empleadas, se observó reflujo ureteral, ni tampoco datos de estenosis de las anastomosis ureterales. Probablemente en relación a la técnica antirreflujo empleada y el ser esta derivación un reservorio de baja presión, no se observaron complicaciones en relación a infecciones urinarias-pielonefritis por lo menos en mayor proporción a lo habitual o que precisasen mayor número de ingresos hospitalarios. (Figs. 4 y 5).

A modo de conclusión, la urocolostomía ha resultado ser una derivación única, de escasa utilización, y muy útil en determinadas situaciones concretas ${ }^{14,15}$. Dicha derivación evita una doble ostomía (colostomía más ostomía urinaria), en pacientes sometidos a cirugía en determinadas patologías. La técnica antireflujo empleada evitó el desarrollo de reflujo ureteral debido a incompetencia de los orificios ureterales y secundariamente las infecciones urinarias. A ello también contribuye que el colon abocado a una ostomía cutánea se comporta como un reservorio de baja presión, evitando así un posible reflujo debido a altas presiones. En todos los casos la urocolostomía se mantuvo normofuncionante al igual que la función renal a lo largo de los distintos seguimientos. La adaptación de los pacientes a una única ostomía fue buena, no excluyendo una futura reconstrucción. 


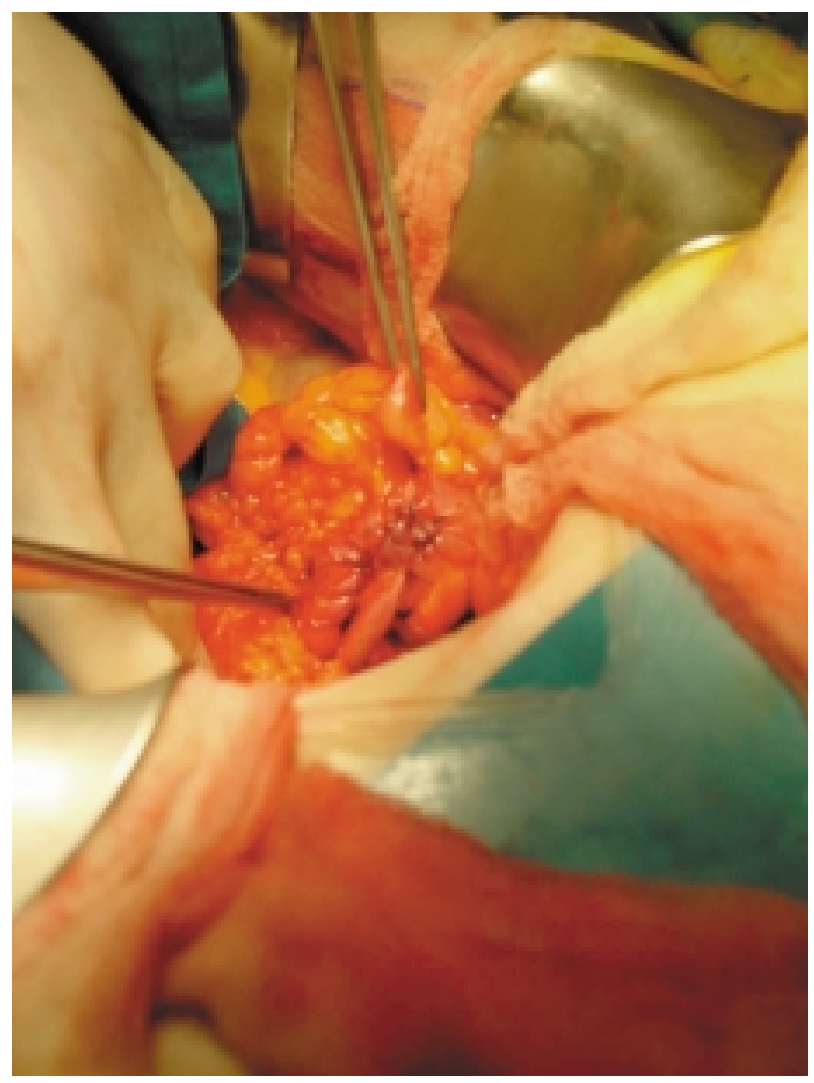

FIGURA 2. Uréteres abocando a colon.

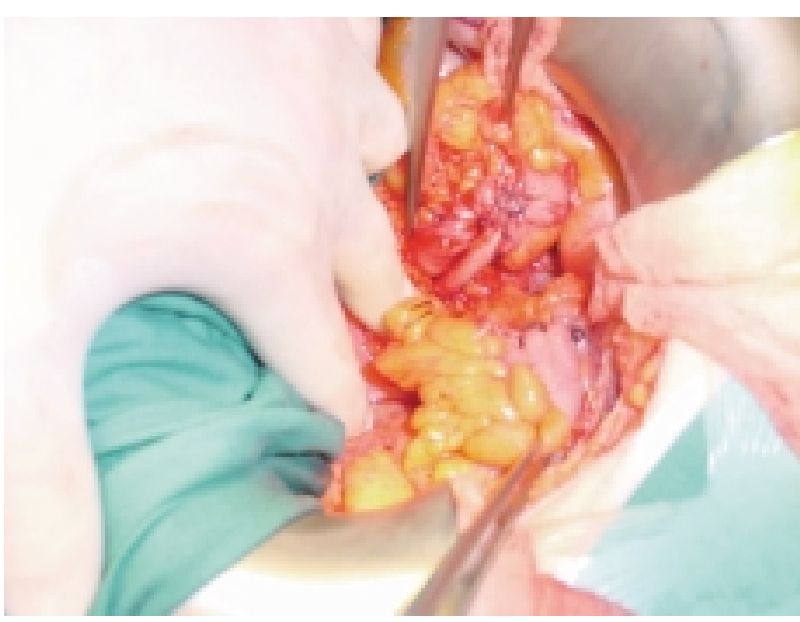

FIGURA 3. Uréter abocando a colon y muñon colónico distal.

\section{REFERENCIAS}

1. BRUNSCHWIG A.: Complete excision of pelvic viscera for advanced carcinoma: one stage abdominal operation with end colostomy and bilateral ureteral implantation into colon above colostomy. Cancer 1948; 1: 177-180.

2. BRUNSCHWIG A, DANIEL W.: Pelvic exenteration operations: with summary of sixty-six cases surviving more than five years. Ann Surg 1960; 151: 571-574.

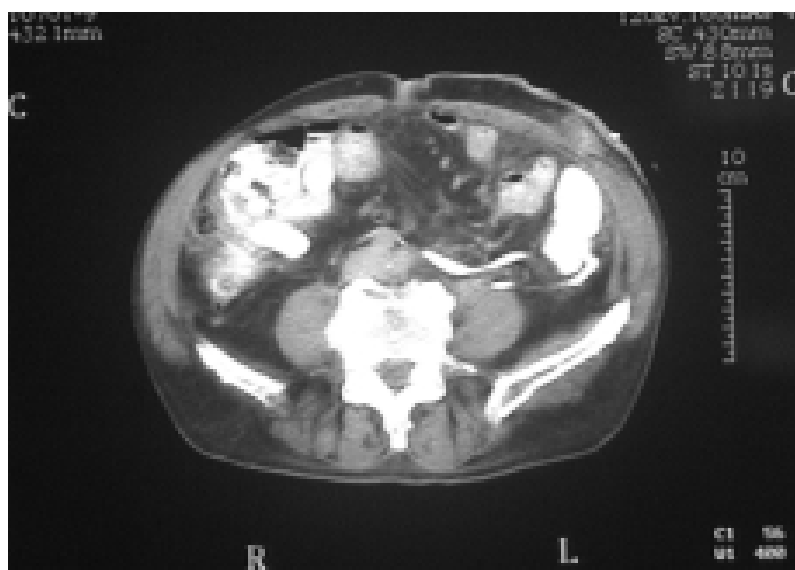

FIGURA 4. T.A.C. con contraste: se observan los dos uréteres contrastados desembocando en el asa colónica distal.

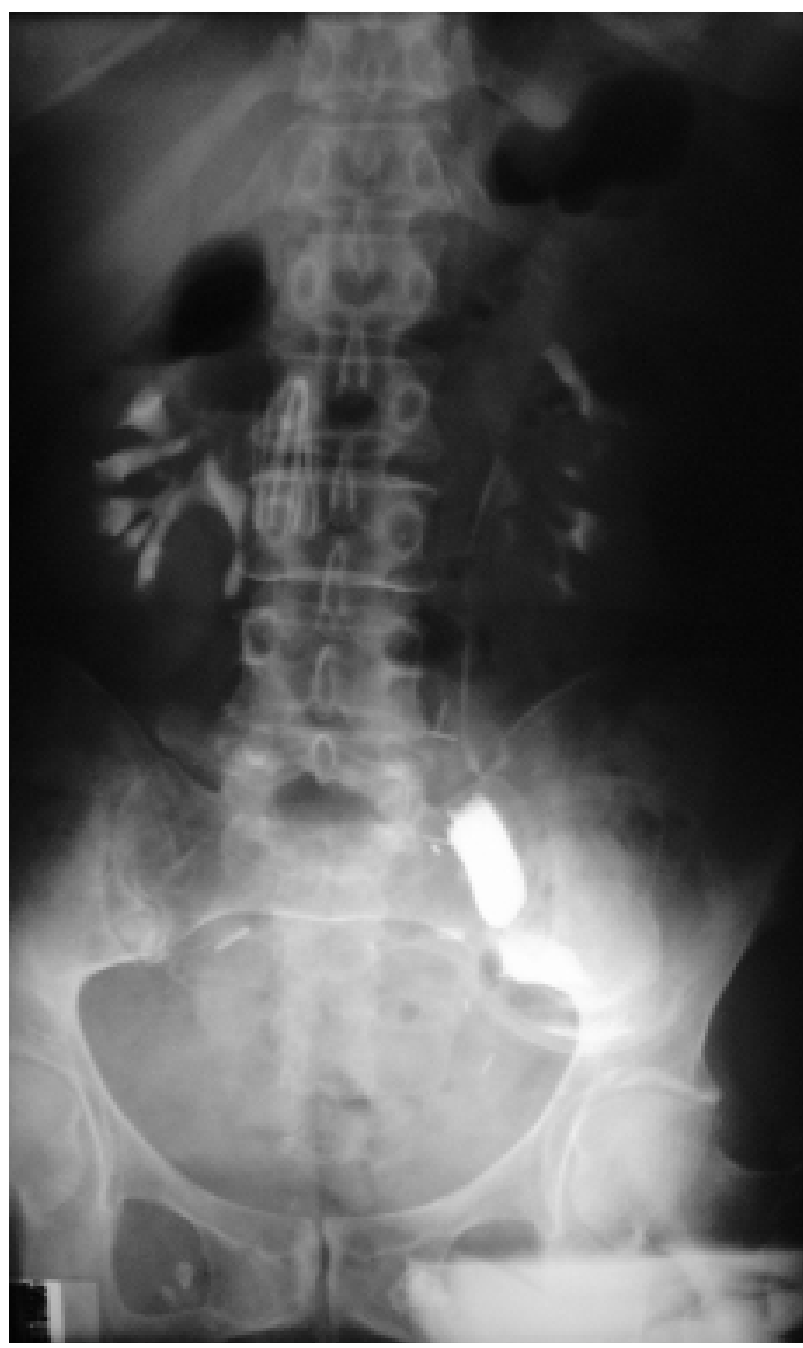

FIGURA 5. Urografia intravenosa: observamos dos sistemas pélvico caliciales de buen aspecto, sin datos de dilatación. Se observa asa de colostomía contrastada. 
3. CARTER MF, DALTON DP, GARNETT JE.: Simultaneous diversion of the urinary and fecal streams utilizing a single abdominal stoma: the double barred wet colostomy. J Urol 1989; 141: 1189-1191.

4. CARTER MF, DALTON DP, GARNETT JE.: The double barred wet colostomy: long-term experience with the first 11 patients. $J$ Urol 1994; 152: 2312-2315.

5. DAVIS BE, NOBLE MJ.: Simplified urinary diversion in patients with preexisting or inminent colostomy. J Urol 1992; 147: 1245-1247.

6. RICHIE JP, SKINNER DG, WAISMAN J.: The effect of reflux in the development of pyelonephritis in urinary diversion: an experimental study. $J$ Surg Res 1974; 16: 256-259.

7. GITTES RF.: Carcinogénesis in ureterosigmoidostomy. Urol Clin N Amer 1986; 13: 201-203.

8. CHIANG MS, MINTON JP, CLAUSEN K, CLATWORTHY HW, WISE HA.: Carcinoma in a colon conduit urinary diversion. J Urol 1982; 127: 1185-1188.

9. WILSON JW, MORALES A.: Development of adenocarcinoma in transverse colon conduit. Urology 1982; 20: 182 .

10. TREIGER BFG, MARSHALL FF.: Carcinogénesis and the use of intestinal segments in the urinary tract. Urol Clin N Amer 1991; 18: 737-740.
11. GITTES RF.: Ureterosigmoidostomy: the pros and cons. Dial Ped Urol 1982; 5: 6-8.

12. RICHIE JP.: Nonrefluxing sigmoid conduit for urinary diversion. Urol Clin N Amer 1979; 6: 469-471.

13. SCHMIDT JD, BUCHSBAUM HJ.: Transverse colon conduit diversion. Urol Clin N Amer 1986; 13: 233237.

14. OSORIO GULLON A, DE OCA J, LÓPEZ COSTEA MA et al.: Double barred wet colostomy: a safe and simple method after pelvic exenteration. Int $J$ Colorrectal Dis 1997; 12: 37-41.

15. MARTÍN OSORIO JA, PIRO BIOSCA C, DE DIEGO ÁlVAREZ M, GOSÁlBEZ JORDA R.: Colonic conduit: an alternative to the treatment of urinary incontinence. Cir Pediatr 1996; 9: 163-165.

Dr. A. Blanco Díez

C/ Montserrat, 18 - 6ํㅡ. E. (Eirís)

15009 La Coruña

(Trabajo recibido el 19 mayo de 2003) 